\title{
Análisis de los determinantes de revelación de instrumentos financieros mediante el uso de componentes principales: el caso de empresas mexicanas*
}

doi:10.11144/Javeriana.cc17-44.adri

\section{Noemí Vásquez-Quevedo}

Profesora investigadora del Departamento de Finanzas y

Contabilidad, Tecnológico de Monterrey, Campus Ciudad

de México. Doctora en administración.

Correo electrónico: nvasquez@itesm.mx

\section{José Jorge Mora-Rivera}

Profesor investigador del Departamento de Economía

y Negocios Internacionales, Tecnológico de Monterrey,

Campus Ciudad de México. Doctor en economía.

Correo electrónico: jjmora@itesm.mx

\footnotetext{
* Artículo de investigación científica y tecnológica. Este artículo es producto del proyecto de investigación Análisis de los determinantes de revelación de instrumentos financieros mediante el uso de componentes principales: el caso de empresas mexicanas, realizado en 2014-2015, cofinanciado por el Tecnológico de Monterrey, Campus Ciudad de México.
} 
Resumen El propósito de esta investigación es identificar las principales características que determinan el nivel de cumplimiento de la IFRS 7 (Instrumentos financieros: información a revelar) para un conjunto de empresas mexicanas. Con la técnica de componentes principales para medir el índice de revelación de las empresas, los resultados econométricos destacan que el tipo de auditor, el nivel de multinacionalidad y, en menor medida, el tamaño de las empresas son los determinantes clave para que las empresas cumplan la norma internacional de información financiera relativa a la revelación de instrumentos financieros. Estos hallazgos son consistentes con los obtenidos con métodos previos para medir el índice de revelación, lo que genera robustez y consistencia a nuestros resultados.

Palabras clave Componentes principales; índice de revelación; instrumentos financieros; NIIF

Códigos JEL M400, M480

\section{Analysis of Financial Instrument Disclosure Determinants by Means of Main Component Analysis: The Case of Mexican Companies}

\footnotetext{
Abstract The purpose of this research is to identify the main characteristics that determine the compliance level of IFRS 7 (Financial instruments: disclosures) for a group of Mexican companies. Using the main components technique to measure the disclosure index of companies, the econometric results point out that the type of auditor, the multinationality level and, to a lesser extent, the size of companies are the key determinants for companies to comply with the International Financial Reporting Standard regarding the disclosure of financial instruments. These findings are consistent with those obtained with previous methods to measure the disclosure index, which gives robustness and consistency to our results.
}

Keywords main components; disclosure index; financial instruments; IFRS

\section{Análise das determinantes de divulgação de instrumentos financeiros mediante o uso de componentes principais: o caso de empresas mexicanas}

Resumo O propósito desta pesquisa é identificar as principais caraterísticas que determinam o nível de cumprimento da IFRS 7 (Instrumentos financeiros: informação a divulgar) para um conjunto de empresas mexicanas. Com a técnica de componentes principais para medir o índice de divulgação das empresas, os resultados econométricos destacam que o tipo de auditor, o nível de multinacionalidade e, em menor medida, o cumprimento das empresas são as determinantes chaves para as empresas cumprir com a norma internacional de informações financeiras relativa à divulgação de instrumentos financeiros. Estes achados são consistentes com os obtidos com métodos prévios para medir o índice de divulgação, gerando robustez e consistência nos nossos resultados.

Palavras-chave Componentes principais; índice de divulgação; instrumentos financeiros; NIIF

\section{Introducción}

La creciente tendencia hacia la globalización demanda que los mercados de capitales sean cada vez más homogéneos y con ello, también aumenta la necesidad de los inversionistas de información elaborada con estándares de información financiera armonizados (Zaidi \& Paz, 2015). La visión de una normatividad contable global ha sido apoyada públicamente por muchas organizaciones internacionales, entre las cuales están el G-20, el Banco Mundial, el Fondo Monetario Internacional, el Comité de 
Basilea, la Organización Internacional de Comisiones de Valores y la Federación Internacional de Contadores (IFRS, s.f.).

Ante la progresiva adopción de las Normas Internacionales de Información Financiera (IFRS, por sus siglas en inglés) en diversos países, la investigación académica sobre el tema también se ha acrecentado. Los primeros estudios analizaban el impacto de la adopción de las Normas Internacionales de Contabilidad, antecesoras de las IFRS, en diferentes países. Estos estudios, en su mayoría empíricos, han analizado los resultados de utilizar las IFRS mediante su impacto en la relevancia valorativa, la calidad de los reportes financieros y el nivel de cumplimiento de revelación de información; siendo estos principalmente en países de Europa y Asia, y en menor medida en África y América (Chua \& Taylor, 2008; Gebhardt, 2012; Lopes \& Rodrigues, 2007; Zaidi \& Paz, 2015).

En esta oleada de adopción de las IFRS, México no ha sido la excepción. Desde la creación del Consejo Mexicano de Normas de Información Financiera (CINIF) en 2003, el país está en el proceso de homologar la normatividad contable local con las IFRS; a partir de 2012, las empresas emisoras de valores están obligadas a implementarlas. Ante este hecho, resulta interesante revisar los primeros resultados de tal adopción, en cuanto al cumplimiento de la normatividad internacional de instrumentos financieros, que ha sido una de las más controversiales por más de dos décadas (Gebhardt, 2012), en específico, de la IFRS 7 Instrumentos financieros: información a revelar.

El objetivo del estudio es identificar las características que impulsan a las empresas mexi- canas a proporcionar más y mejor información sobre instrumentos financieros en los reportes anuales entregados a la institución reguladora, en este caso, la Comisión Nacional Bancaria y de Valores (CNBV) y, por ende, cumplir de manera adecuada con la implementación de las IFRS en México. Hay una vasta literatura sobre el estudio de la revelación financiera que abarca temas que van desde el estudio de teorías que explican los incentivos de divulgación de información (Gray, Meek \& Roberts, 1995; Hossain, Tan \& Adams, 1994; Lang \& Lundholm, 1993); estudios empíricos (Chow \& Wong-Boren, 1987; Cooke, 1989, 1991; Jensen \& Meckling, 1976); hasta el estudio de factores de divulgación de acuerdo con lo señalado en alguna IFRS (Devalle \& Rizzato, 2012; Lopes \& Rodrigues, 2007).

La presente investigación propone una forma alternativa de obtener el índice de revelación con el método de componentes principales (MCP). Investigaciones previas han utilizado indicadores sintéticos que consisten en un enfoque dicotómico al sumar los criterios establecidos que debe cumplir cada empresa. Lo anterior ya sea al realizar una suma ponderada o al discriminar los criterios de no aplicabilidad (Tsalavoutas, Evans \& Smith, 2010).

Por su parte, el método empleado en este trabajo es una de las técnicas más tradicionales de análisis multivariado (Hotelling, 1933) y ha sido empleada en diversos contextos para el estudio de las empresas. Por ejemplo, para determinar e interpretar la eficiencia en la gestión administrativa (Rescala, Devincenzi, Rohde, Bonaffini, Giraudo, Bernaola \& Pavón, 2012); en la realización de análisis económicos y financieros ante la puesta en marcha de programas 
gubernamentales (García-Fernández, López-Díaz \& González-Díaz, 2008); y en la modelación estadística y computacional para el diagnóstico y el establecimiento de estrategias de análisis de sostenibilidad de las empresas (Vázquez-Alfonso, Guerra-Bustillo \& Sánchez-León, 2014). La idea básica del método consiste en describir la varianza de un conjunto de datos multivariados tan parsimoniosamente como sea posible, utilizando un conjunto de variables derivadas no correlacionadas, cada una de las cuales es una combinación lineal particular de las variables del conjunto original de datos (Everitt \& Dunn, 2001).

El interés de generar indicadores cada vez más adecuados ha estimulado a que en años recientes distintos organismos nacionales e internacionales se hayan dado a la tarea de construir indicadores sintéticos para diversos fines y propósitos, como la evaluación de políticas sociales, la focalización de ayuda a países vulnerables (PNUD, 2014), la medición del grado de consecución de objetivos globales, el asesoramiento en los procesos de planificación y la evaluación de la calidad de los reportes corporativos, entre otros (UNCTAD, ISAR, 2014).

La elaboración de cada uno de estos indicadores responde a los objetivos de cada estudio y a la disposición de información para su realización. Los procedimientos de obtención de los indicadores sintéticos se diferencian fundamentalmente por la forma en la que se ponderan y agregan los indicadores del sistema original. La metodología de componentes principales en el presente estudio ha sido utilizada y probada en diferentes escenarios, pese a ello aún no ha sido empleada en contextos como el de esta investigación; es decir, no se tiene evidencia de que esta técnica haya sido usada en la construcción de un indicador de revelación financiera y más aún para el caso específico de las empresas mexicanas.

Las contribuciones concretas de esta investigación, que se suman a los hallazgos de estudios previos, consisten en: 1) utilizar datos de las empresas mexicanas y determinar las variables que influyen en mayor medida en el cumplimiento de la IFRS 7, ejercicio que aún no se realiza para el caso mexicano; 2) emplear una técnica multivariada para la generación del índice de revelación que permite aprovechar la heterogeneidad de la información que las empresas reportan; y 3) contrastar los resultados alcanzados con otras formas de medir el índice de revelación que permite efectuar un ejercicio de robustez a nuestros hallazgos (Cotter, Lokman \& Najah, 2011; Lopes \& Rodrigues, 2007).

El artículo está organizado como sigue. El primer apartado presenta la revisión de la literatura sobre revelación financiera y los efectos de los estándares contables de instrumentos financieros en la administración del riesgo y en los reportes financieros. El segundo apartado presenta el diseño de la investigación, identificando la muestra, las variables del estudio, las hipótesis a contrastar y las estadísticas descriptivas. La tercera sección describe el modelo econométrico y la interpretación de los resultados. En la sección final se exponen las conclusiones.

\section{Revisión de la literatura}

Hemos dividido la revisión de la literatura en dos grupos, debido al amplio campo de estudio 
que esta representa en la investigación contable. En el primero, presentamos los estudios relacionados con los determinantes de las prácticas de divulgación, basados en las características de la empresa y su relación con diferentes teorías. En el segundo, revisamos investigaciones referentes a los efectos del uso y nivel de cumplimiento de normas contables, en particular las relacionadas con los instrumentos financieros.

\subsection{Determinantes de revelación}

Ante la creciente demanda de información financiera de alta calidad que pueda generar confianza para todos los usuarios en general, y para los inversionistas potenciales en particular, las empresas utilizan la revelación como una herramienta valiosa para proporcionar información por medio de las notas en los estados financieros. En respuesta a esta demanda, surgen trabajos como el de Alan Robert Cerf (1961), que inició el análisis de los atributos específicos que determinan el nivel de revelación de información de las empresas. A partir de entonces, la literatura sobre revelación de información ha sido una de las más amplias en la investigación contable.

La revelación como medio de comunicación de información económica ha sido estudiada como de emisión obligatoria (Devalle \& Rizzato, 2012; Popova, Georgakopoulos, Sotiropoulos \& Vasileiou, 2013; Tsalavoutas, Evans \& Smith, 2010) y de emisión voluntaria (Chow \& Wong-Boren, 1987; Cooke, 1991; Jensen \& Meckling, 1976). R. S. Olusegun Wallace y Kamal Naser (1995) consideran que la primera es responsabilidad de los organismos reguladores, como la International Accounting Standards Board, IASB y la Financial Accounting Standards Board, FASB, y la segunda es responsabilidad de la gerencia de la empresa para proveer información adicional acerca del valor de la compañía y del desempeño de sus administradores.

Por otro lado, la literatura ha sido acrecentada por el estudio de distintas teorías que explican los incentivos para divulgar información, como los costos de agencia (Hossain, Tan \& Adams, 1994), costos de transacción (King, Pownall \& Waymire, 1990), costos políticos (Cahan, 1992), costos de propiedad (Verrecchia, 1983), costos de emisión de información (Lang \& Lundholm, 1993), influencias culturales (Zarzeski, 1996) y presiones de los mercados de valores (Gray, Meek \& Roberts, 1995).

En particular, la teoría de agencia se ha utilizado en la literatura contable para explicar las prácticas de revelación en varios países y es el marco de referencia para el desarrollo del análisis empírico. Por ello, en el presente estudio se utiliza buena parte de las variables que captan la construcción de tal teoría: el tamaño de la empresa, apalancamiento, cotización en el extranjero y tipo de auditor (Abd-Elsalam \& Weetman, 2003; Chalmers \& Godfrey, 2004; Chow \& Wong-Boren, 1987; Cooke, 1991; Jensen \& Meckling, 1976; Lopes \& Rodrigues, 2007; Tarca, Morris \& Moy, 2005; Watts \& Zimmerman, 1978, 1986; Zarzeski, 1996).

Otras variables utilizadas en esta investigación son multinacionalidad (Cooke, 1989), gobierno corporativo (Doupnik \& Salter, 1995; Fechner \& Kilgore 1994), adopción anticipada de las IFRS (Zehri \& Chouaibi, 2013) 
y sostenibilidad (Bebbington, Larrinaga-González \& Moneva-Abadía, 2008).

\subsection{Revelación de instrumentos}

\section{financieros}

La contabilidad de instrumentos financieros es uno de los temas más controversiales en la normatividad contable, sobre todo en cuanto a su medición a valor razonable. Gran parte de la investigación en torno a este tema está enfocada en la industria financiera y utiliza datos de revelación de empresas estadounidenses de la década de los 90. Por consiguiente, para empresas no financieras hay poca evidencia disponible sobre el uso de instrumentos financieros y los efectos de sus estándares contables (Gebhardt, 2012).

Günther Gebhardt (2012) presenta el porcentaje de instrumentos financieros respecto del total de activos financieros y del total de activos, que son medidos a valor razonable utilizando los tres niveles de jerarquía, de acuerdo con lo señalado en la IFRS 7. Sus resultados muestran que las empresas hacen uso en buena medida del valor razonable; pese a ello, señala que aún hay amplias oportunidades en la investigación empírica futura sobre el tema.

Las líneas de investigación en torno a los efectos de los estándares contables de instrumentos financieros se pueden clasificar en tres: la relacionada con los efectos en la administración del riesgo financiero (Lins, Servaes \& Tamayo, 2011; Zhang, 2009); la correspondiente a los efectos sobre el flujo de efectivo, utilidades y administración de utilidades (Bodnar \& Gebhardt, 1999; Dechow, Myers \& Shakespeare, 2010; Zhang, 2009) y la referente a los efectos en la relevancia de los estados financieros (Chambers, Linsmeier, Shakespeare \& Sougiannis, 2007; Song, Thomas \& Yi, 2010).

El trabajo de Patrícia Teixeira Lopes y Lúcia Lima Rodrigues (2007) mezcla los efectos de aplicar normatividad contable relativa con instrumentos financieros y las características de las firmas con mayor nivel de revelación. Otros estudios que cuantifican el grado de cumplimiento con alguna IFRS en particular, utilizando un índice de revelación, incluyen los de Omneya H. Abd-Elsalam y Pauline Weetman (2003), Keryn Chalmers y Jayne M. Godfrey (2004), Martin Glaum y Donna L. Street (2003), Donna L. Street y Stephanie M. Bryant (2000), Donna L. Street y Sidney J. Gray (2001).

\section{Diseño de la investigación}

Es esta sección se describe la muestra de las empresas, las variables del modelo econométrico, las hipótesis a contrastar y algunos rasgos de las estadísticas descriptivas de la base de datos.

\subsection{Selección de la muestra y variables empleadas}

La muestra se conforma de 93 empresas que no pertenecen al sector financiero y estaban listadas en la Bolsa Mexicana de Valores (BMV) en 2012 (anexo 1). Estas empresas cuentan con estados financieros de conformidad con las IFRS y con reportes anuales, en cuyo contenido están disponibles notas de revelación. Tales empresas están obligadas a presentar su información financiera de conformidad con las IFRS desde 2012 (CNBV, 2008), período del presente estudio. 
La tabla 1 presenta las empresas analizadas por sector económico. El sector que contribuye con mayor proporción es el de las manufacturas (22,6\% de la muestra), el 18,3\% pertenece al sector minería, seguido por el sector comercio con 16,0\%. Del sector de la construcción se cuenta con información de 11 empresas, mien- tras que el sector de comunicaciones y transportes participa con 10 empresas del total. Vemos que esta conformación de la muestra recoge buena parte de los sectores económicos presentes en la economía mexicana, a excepción del sector primario (agricultura, ganadería, silvicultura y pesca) que solo representa el 2,2\%.

\begin{tabular}{|c|c|c|}
\hline Sector económico & $\mathbf{N}$ & Porcentaje de la muestra (\%) \\
\hline Manufacturas & 21 & 22,6 \\
\hline Minería & 17 & 18,3 \\
\hline Comercio & 15 & 16,1 \\
\hline Construcción & 11 & 11,8 \\
\hline Comunicaciones y transporte & 10 & 10,8 \\
\hline Agricultura, ganadería, silvicultura y pesca & 2 & 2,2 \\
\hline Otros & 17 & 18,3 \\
\hline Total & 93 & 100,0 \\
\hline
\end{tabular}

Tabla 1

Distribución de las empresas por sector económico

Fuente: elaboración propia con información de Economática

\section{A. Variable dependiente}

Con el objetivo de identificar el cumplimiento de requisitos de revelación señalados en la IFRS 7 (IFRS, 2014) y de construir el índice de revelación con el MCP, se aplicó la técnica de análisis de contenido a los reportes anuales de las compañías (Oliveira, Rodrigues \& Craig, 2006), para determinar un índice de calificación de acuerdo con lo que señala esta norma.

A partir de una revisión exhaustiva de los requerimientos señalados en la IFRS 7, se identificaron siete categorías de información de acuerdo con los temas más importantes de la norma y que varios de ellos han sido considerados en otros estudios que revisan la aplicación de estándares contables sobre ins- trumentos financieros (IAS 32, IAS 39)

(Gebhardt, 2012; Lopes \& Rodrigues, 2007).

$\mathrm{Al}$ analizar el cumplimiento en cada una de estas categorías, se asignó un valor de 1 a aquellos criterios de la IFRS 7 que sí cumplía cada empresa, y un valor de cero para el incumplimiento (Cooke, 1992). En adición al criterio dicotómico de calificación, se eliminaron de la muestra los requerimientos que el 100\% de las empresas cumple y aquellos que no le son de aplicación a más del 90\%. De esta forma, quedaron 26 criterios integrados en las siguientes categorías (entre paréntesis \# de criterios) que se pueden identificar en el anexo 2 :

1. Categorías de instrumentos financieros (4)

2. Valor razonable (7) 
3. Riesgos (generalidades) (3)

4. Riesgo de crédito (2)

5. Riesgo de liquidez (2)

6. Riesgo de mercado (2)

7. Estado de resultados integral (6)

Con una base de datos restringida fue posible aplicar el MCP y generar la variable dependiente (índice de revelación) propuesta en la presente investigación. El procedimiento consistió en partir de un vector $x$ dado, que representa la información de cumplimiento de las empresas analizadas, así la primera componente principal de las observaciones, $y$, es la siguiente combinación lineal:

$y=a_{1}\left(\frac{x_{1}-\overline{x_{1}}}{s_{1}}\right)+a_{2}\left(\frac{x_{2}-\overline{x_{2}}}{s_{2}}\right)+\ldots+a_{p}\left(\frac{x_{p}-\overline{x_{p}}}{s_{p}}\right)$

cuya varianza muestral es la más grande entre todas las combinaciones lineales, sujeta a la restricción $a a^{\prime}=1$, donde $a=\left(a_{1}, a_{2}, \ldots, a_{p}\right)^{\prime}$ es el vector de coeficientes, y $\bar{x}_{k}$ y $s_{k}$ son la media y la desviación estándar de cada variable $x_{k}$. La calificación de componentes principales para la empresa $i$ con vector de cumplimiento $x_{i}$ es entonces $y_{i}=a^{\prime} \tilde{x}_{l}$ donde $\tilde{x}_{\underline{l}}$ es el vector de variables estandarizadas $\frac{x_{k}-x_{k}}{s}$. Esta variable transformada tiene media cero y varianza $\lambda$, donde $\lambda$ es el eigenvalor más grande de la matriz de correlación de $x$.

Por lo tanto, la primera componente principal nos da un índice que proporciona la máxima discriminación entre las empresas, con los criterios de cumplimiento que varían más entre las empresas que tienen un peso mayor. Por ejemplo, un criterio que la mayoría de las empresas cumpla tendrá un peso prácticamente nulo en la primera componente principal, ya que contribuye poco a la variación entre estas, de manera similar sucede para un criterio que no cumpla ninguna empresa. Desde la perspectiva de la medición del nivel de cumplimiento relativo, hay aún más razón para favorecer el uso de los componentes principales, ya que los componentes principales explícitamente dan un peso mayor a las variables de cumplimiento que están distribuidas de manera más heterogénea entre las empresas.

Para cada empresa $i$ es posible calcular la calificación del primer componente principal, $y_{i}$, el cual se toma como una variable proxy para el nivel de cumplimiento o revelación de información financiera de las empresas.

\section{B. Variables independientes e hipótesis}

\section{de investigación}

Las variables independientes representan las características de las empresas que se consideran determinantes potenciales de la revelación de información. Tales variables tienen su fundamento en investigaciones previas y, en conjunto con las hipótesis de nuestra investigación, son descritas a continuación.

\section{Variables independientes}

\section{Tamaño}

Varios estudios de revelación sugieren una relación positiva entre el tamaño de la firma y la extensión de revelación financiera voluntaria (Chow \& Wong-Boren, 1987; Cooke, 1989, 1991; Jensen \& Meckling, 1976). Ross L. Watts y Jerold L. Zimmerman $(1978,1990)$ y Steven 
F. Cahan (1992) consideran que la visión política de la empresa hacia el futuro, causada por el costo político de generar confianza de los inversionistas y reducir la carga fiscal, es otra razón potencial de tal relación positiva.

Por otro lado, la acumulación de información es costosa, de tal forma que el aspecto económico influye en la revelación financiera de las firmas (Lang \& Lundholm, 1993). Las firmas grandes tienden a estar listadas en una bolsa de valores y tienen la motivación de revelar más información para mantener la demanda de sus acciones (Gray, Meek \& Roberts, 1995); además los inversionistas pueden interpretar la falta de información como señal de "mala noticia" y afectar el valor de la firma (Hossain, Tan \& Adams, 1994).

Esta variable es medida con dos aproximaciones: el logaritmo natural del total de activos y el logaritmo natural del total de ventas netas.

$H_{1}$ : Las empresas de gran tamaño presentan un mayor nivel de revelación de información sobre instrumentos financieros que las empresas de menor tamaño.

\section{Cotización en el extranjero}

La relación entre la empresa y su cotización en el extranjero se basa en los costos de agencia (Jensen \& Meckling, 1976) y en la teoría de señales (Ross, 1977). Los costos de agencia incluyen los de incentivación, supervisión, costos de garantía y los de pérdidas residuales. Por otro lado, la teoría de señales afirma que la composición del financiamiento de una empresa está definida por las características propias de esta y no por el tipo de financiamiento por el que se opte. Por lo tanto, si una compañía está listada en bolsas de valores distintas a la de origen, sus costos de agencia serán mayores. Este argumento tiene su base en el trabajo de Terry E. Cooke (1989), quien sustenta que la compañía desea aumentar su capital en los mercados extranjeros.

Esta variable dicotómica toma un valor de 1 si la empresa se encuentra listada en la BMV y en una bolsa de valores extranjera, y un valor de 0 si solo cotiza en México.

$\mathrm{H}_{2}$. Las compañías que cotizan en la BMV y en alguna bolsa de valores extranjera tienen un grado de revelación de información sobre instrumentos financieros mayor que las compañías que solo cotizan en la BMV.

\section{Multinacionalidad}

De acuerdo con Timothy Doupnik y Hector Perera (2012), las diferencias en la contabilidad de las empresas pueden generar distorsiones en sus estados financieros consolidados y dificultar la comparabilidad de los mismos entre las subsidiarias de una misma matriz, sobre todo cuando actúan en países con sistemas contables distintos. Por otro lado, Terry E. Cooke (1989) afirma que las compañías que operan en más de un país tienden a controlar mejor su administración, debido al grado de complejidad de sus operaciones.

Esta variable es medida dividiendo el total de ventas en el extranjero entre las ventas totales de la compañía.

$H_{3}$. Las empresas con un mayor índice de multinacionalidad presentan mayor nivel de revelación de información sobre instrumentos financieros. 


\section{Apalancamiento}

Omneya H. Abd-Elsalam y Pauline Weetman (2003) y Marilyn Taylor Zarzeski (1996) sustentan la existencia de una relación negativa entre el apalancamiento y la revelación de información. Sus argumentos se basan en la teoría de señales, que afirma que el endeudamiento es una de las mejores opciones que posee una empresa para financiarse y en el hecho de que las compañías con mayor apalancamiento pertenecen a empresas financieras, las cuales prefieren mantener mayor información privada. Como contraargumento, Ann Tarca, Richard D. Morris y Melissa Moy (2005) plantean que las empresas con mayor financiamiento externo usan normas contables como las IFRS.

La variable se mide dividiendo el total de pasivos entre el total de capital contable.

$\mathrm{H}_{4}$. Las empresas con un mayor apalancamiento presentan un mayor grado de revelación de información sobre instrumentos financieros.

\section{Tipo de auditor}

De acuerdo con Keryn Chalmers y Jayne M. Godfrey (2004), para mantener una reputación impecable, las grandes empresas buscan ser auditadas por alguna de las firmas internacionales de auditoría más prestigiosas y conocidas como las Big 4. Las grandes firmas auditoras poseen mayor conocimiento de los estándares internacionales de contabilidad y, por lo tanto, los costos de implementarlos y auditarlos son menores para las Big 4 que para empresas auditoras de menor tamaño.

Michael C. Jensen y William H. Meckling (1976) y Ross L. Watts y Jerold L. Zimmerman (1986) consideran que los auditores juegan un papel importante al limitar el comportamiento oportunista por parte de la gerencia, lo que reduce los costos de agencia a cargo de directores y gerentes (agentes).

Esta variable dicotómica toma el valor de 1 si la compañía es auditada por alguna de las Big 4 y un valor de 0 en caso contrario. Según Toscano-Moctezuma y García-Benau (2012), este grupo de firmas está conformado por Deloitte, PricewaterhouseCoopers (PwC), Ernst Young (EY) y KPMG.

$H_{5}$. Las empresas que presentan estados financieros auditados por alguna de las firmas Big 4 muestran un grado de revelación de información sobre instrumentos financieros más elevado que las empresas que no son auditadas por alguna de ellas.

\section{Anticipada en la adopción de IFRS}

Fatma Zehri y Jamel Chouaibi (2013) señalan que un país con apertura económica al mercado exterior tiende a adoptar de forma más rápida las IFRS. Por tal motivo, la evolución de los mercados globales de capital, la CNBV comunicó la posibilidad de que las emisoras que así lo desearan y cumplieran ciertos requisitos, podían adoptar las IFRS a partir del ejercicio 2008, lo que se considera como "adopción anticipada" (CNBV, 2008).

Ante la existencia de empresas que tomaron esta iniciativa, se considera relevante incluir la variable de adopción anticipada para encontrar evidencia de que esta característica tenga una repercusión en el nivel de revelación sobre instrumentos financieros.

La variable es dicotómica y toma el valor de 1 si la empresa se anticipó a la implementación de las IFRS y 0 en caso contrario. Con esta premisa, se plantea la hipótesis 6 de la siguiente manera: 
$H_{6}$. Las empresas que adoptaron anticipadamente las IFRS presentan un mayor grado de revelación de información sobre instrumentos financieros que aquellas que no lo hicieron.

\section{Gobierno corporativo}

En México, la Ley del Mercado de Valores (México, 2014) establece que: "las compañías públicas deberán de tener un consejo de administración que no exceda más de 21 consejeros propietarios, de los cuales el 25\% debe calificar como consejeros independientes". La razón de esta regulación es que sean los miembros independientes quienes ejerzan una influencia significativa sobre las decisiones, para contribuir así al desarrollo de mejores estrategias para las empresas. El 98\% de las empresas analizadas cumple la normativa establecida en la LMV.

La teoría de la contingencia, de acuerdo a Timothy Doupnik y Stephen B. Salter (1995) y Harry E. Fechner y Alan Kilgore (1994), indica que la estructura de gobierno corporativo de las empresas puede estar relacionada con las prácticas de revelación de información. La premisa que maneja la teoría de agencia planteada por Roszaini Haniffa y Terry E. Cooke (2002) es que se necesitan consejeros independientes a la empresa para monitorear y controlar las acciones de los gerentes ejecutivos. En conclusión, se espera que aquellas empresas que cumplan el porcentaje mínimo de consejeros independientes, cuenten con un nivel de revelación mayor al de las empresas que tengan un consejo familiar.

Esta variable es medida con el porcentaje de miembros independientes que integran los consejos de administración.
$H_{7}$. Las empresas con un mayor cumplimiento de gobierno corporativo presentan un mayor grado de revelación de información sobre instrumentos financieros.

\section{Sostenibilidad}

Diversas compañías revelan su desempeño en materia de sostenibilidad como una forma de controlar el riesgo de su reputación como lo mencionan Jan Bebbington, Carlos LarrinagaGonzález y José M. Moneva-Abadía (2008) y Sidney J. Gray, Gary K. Meek y Clare B. Roberts (1995), que afirman que la revelación de sostenibilidad sirve para facilitar la proyección de una imagen social-contable. La revelación de esta información incrementa la legitimidad y al mismo tiempo le permite a la compañía manejar los riesgos de su reputación, de acuerdo a Charles J. Fombrun, Naomi A. Gardberg y Michael L. Barnett (2000).

El mercado internacional está promoviendo este tipo de revelación por medio del International Integrated Reporting Council (IIRC, http:// integratedreporting.org/) y el Sustainability Accounting Standards Board (SASB, www.sasb. org), que tienen como objetivo crear un esquema de contabilidad globalmente aceptado que involucre información referente a sostenibilidad junto con información financiera, ambiental, social y de gobierno corporativo (Deloitte, s.f.).

Se trata de una variable dicotómica con valor de 1 si las empresas revelan alguna información sobre sostenibilidad (ejemplo: regulación ambiental, medio ambiente o cambio climático) y 0 si no lo hacen.

$H_{8}$. Las empresas que revelan información sobre el desempeño de la empresa en materia de 
sostenibilidad presentan un mayor grado de revelación de información sobre instrumentos financieros.

\subsection{Estadísticas descriptivas}

La tabla 2 muestra las estadísticas descriptivas de las variables independientes. El valor promedio del total de activos de la muestra es mayor a los 55 millones de pesos mexicanos, con un valor máximo que excede los $\$ 1.000$ millones de pesos mexicanos. En promedio, las ventas netas representan el $80 \%$ del valor total de los activos, con un monto mínimo cercano a los
\$150.000. Poco más del 30\% de las empresas cotiza en el extranjero, mientras que el $87 \%$ es auditado por alguna de las firmas más importantes en el ramo y solo 13\% de las empresas realizó la adopción anticipada de las IFRS, de acuerdo con lo establecido por la CNBV. Además, el 92\% realiza revelaciones sobre sostenibilidad. En promedio, el 46\% de las empresas está integrado por miembros independientes en su consejo de administración, la empresa con la mayor proporción de consejeros independientes registró un $95 \%$.

\begin{tabular}{lrrrr}
\hline \multicolumn{1}{c}{ Variables } & \multicolumn{1}{c}{ Media } & Desviación estándar & Mínimo & \multicolumn{1}{c}{ Máximo } \\
\hline Activo total & $\$ 55.250 .305,00$ & $122.821 .805,00$ & $\$ 321.777,00$ & $\$ 1.002 .569 .285,00$ \\
Ventas netas & $\$ 43.827 .571,00$ & $98.872 .910,00$ & $\$ 148.840,00$ & $\$ 775.069 .645,00$ \\
Apalancamiento & 1,33 & 1,15 & 0,06 & 5,85 \\
Multinacionalidad & 0,19 & 0,25 & 0,00 & 0,85 \\
Gobierno corporativo & 0,46 & 0,17 & 0,13 & 0,95 \\
Tipo de auditor & 0,87 & 0,33 & 0,00 & 1,00 \\
Anticipada & 0,13 & 0,33 & 0,00 & 1,00 \\
Cotiza en el extranjero & 0,32 & 0,47 & 0,00 & 1,00 \\
Sostenibilidad & 0,92 & 0,26 & 0,00 & 1,00 \\
\hline
\end{tabular}

Tabla 2

Estadísticas descriptivas de la muestra

Fuente: elaboración propia con información de los reportes anuales 2012 de la BMV y Economática

\section{Modelo econométrico y resultados}

En esta investigación, utilizamos un modelo de mínimos cuadrados ordinarios (MCO) que considera distintas especificaciones con el objetivo de generar resultados consistentes y robustos. El modelo considera como variable dependiente el índice de revelación ${ }^{1}$ y como variables explica-

1 Para generar contraste y consistencia en los resultados, cinco de las especificaciones utilizan como variable depen- tivas utiliza diversas características exógenas de las empresas señaladas en el apartado anterior.

El modelo econométrico se expresa por medio de la siguiente ecuación, donde el subíndice $i$ hace referencia a cada una de las empresas:

diente el índice de revelación construido mediante el MCP (columnas de los modelos 1-5, en la tabla 3) y dos de las especificaciones empleadas en este análisis utilizan como variable dependiente la proporción de información revelada, generada como el porcentaje de criterios cumplidos dentro del total que debieron cumplir las empresas (columnas de los modelos 4 a y $5 a)$. 
$y_{i}=\beta_{0}+x_{i 1} \beta_{1}+x_{i 2} \beta_{2}+\ldots+x_{i k} \beta_{k}+u_{i}$

En la ecuación (2), $y_{i}$ representa el índice de revelación, las $x_{k}$ las variables explicativas, los $\beta_{k}$ son los coeficientes a estimar y $u_{i}$ representa los errores estocásticos del modelo (Gujarati \& Porter, 2010).

Los resultados de la estimación se presentan en la tabla 3. En las columnas que corresponden a los modelos 1, 2, 3, 4 y 5, la variable dependiente es el índice de revelación construido mediante el MCP, mientras que en las columnas correspondientes a los modelos 4 a y $5 \mathrm{a}$ se utilizó el índice de revelación construido directamente como la proporción del número de características cumplidas dentro del total de las mismas, justo como se realiza en estudios previos (Cooke, 1992; Lopes \& Rodrigues, 2007). Los resultados de estas columnas deben ser vistos como un ejercicio de robustez a nuestros hallazgos centrales que utilizan como variable dependiente el índice de revelación construido mediante el MCP. También se debe señalar que los coeficientes en las columnas de los modelos 5 y 5 a emplean el logaritmo del monto total de ventas de la empresa como variable proxy de su tamaño, lo anterior busca explorar si los resultados encontrados son sensibles a la variable tamaño de la empresa. Se realizó un análisis para detectar problemas de multicolinealidad entre las variables independientes. En el anexo 3 se presenta la matriz de correlaciones de las variables independientes empleadas; en ella se puede apreciar que la correlación parcial entre las variables es baja, el valor más alto está entre las variables valor total de los activos y multinacionalidad, así mismo se puede apreciar que el resto de los valores es bastante bajo, lo que hace suponer que no hay problemas de colinealidad entre los regresores empleados. No obstante y para complementar el argumento anterior, se realizó la prueba conocida como factor de inflación de la varianza (variance inflation factor, VIF) y se detectó que el valor más alto corresponde a la variable logaritmo natural de los activos, VIF = 1,52, lo que nos permite argumentar que no existe multicolinealidad en el modelo ${ }^{2}$. Los resultados de las regresiones incluyen errores estándar robustos para considerar la posible presencia de heterocedasticidad.

En todas las especificaciones se observa que el coeficiente de la variable logaritmo de los activos, como medición del tamaño de la empresa, tiene el signo esperado, aunque en solo tres casos resulta estadísticamente significativa (ver columnas de los modelos $1 \mathrm{al} 4 \mathrm{a}$ ). Este resultado es consistente con lo expuesto por Chee W. Chow y Adrian Wong-Boren (1987) y por Patrícia Teixeira Lopes y Lúcia Lima Rodrigues (2007), que también identifican el tamaño de la empresa como uno de los determinantes de la revelación de instrumentos financieros.

Por otro lado, se observa que la variable relacionada al tipo de auditor resulta significativa en todos los casos; lo que indica, con un alto grado de confiabilidad, que este tipo de control es una característica fundamental para determinar el grado de revelación de informa-

\footnotetext{
2 Detectar valores de VIF superiores a 10 indicaría presencia indiscutible de multicolinealidad en el modelo y sugeriría la exclusión de variables del mismo. En nuestro caso, todos los VIF encontrados son inferiores a 4, valor crítico comúnmente aceptado para detectar o descartar la presencia de multicolinealidad (Gujarati \& Porter, 2010).
} 
ción financiera que caracteriza a las empresas mexicanas. Este resultado es consistente con los estudios de Patrícia Teixeira Lopes y Lúcia Lima Rodrigues (2007); Lídia Oliveira, Lúcia Lima Rodrigues y Russell Craig (2006), e Ioannis Tsalavoutas, Lisa Evans y Mike Smith (2010).

Adicionalmente, resulta primordial señalar que la variable multinacionalidad es significativa en las cuatro especificaciones donde se incluye. Estos resultados permiten evidenciar que cuando se incorpora esta variable, el poder predictivo de las variables relacionadas con el tamaño de la empresa (Ln de activos y Ln de ventas) disminuye de forma considerable. Lo anterior sugiere que, adicional al tamaño de la empresa, un determinante fundamental que influye para que las empresas cumplan con las normas internacionales de información financiera, en particular la IFRS 7, lo constituye el grado de internacionalización de la empresa en cuestión, lo que sugiere que estas empresas deben cumplir las normas de los países en los que tienen relaciones comerciales. Este resultado es distinto a lo encontrado por investigaciones anteriores (Lopes \& Rodrigues, 2007; Oliveira, Rodrigues \& Craig, 2006; Street \& Gray, 2001), seguramente por la forma distinta de cuantificar el índice de revelación, ya que la propuesta de hacerlo en este estudio explota la información en su conjunto, al priorizar aquellos elementos que contribuyen a la identificación de las características heterogéneas que constituyen el índice de revelación de manera primordial.

En términos generales, los resultados muestran evidencia de que las hipótesis $\mathrm{H}_{3}$ y $\mathrm{H}_{5}$ se confirman con un alto grado de confianza estadística. También hay evidencia de que la hipótesis $\mathrm{H}_{1}$, referente al tamaño de la empresa, se confirma en la mayoría de los casos. No hay evidencia estadística que respalde las hipótesis $\mathrm{H}_{2}, \mathrm{H}_{4}, \mathrm{H}_{6}, \mathrm{H}_{7} \mathrm{y} \mathrm{H}_{8}$. Por lo tanto, las variables que determinan el grado de revelación de instrumentos financieros para las empresas mexicanas que cotizan en la BMV son:

1. El índice de multinacionalidad. No se encontraron estudios previos con este resultado; sin embargo, es posible identificar el argumento de Terry E. Cooke (1989) cuando señala que las compañías que operan en más de un país tienden a generar mejores controles en sus procesos administrativos, debido al grado de complejidad de los mismos. Lo anterior sugiere que las diferencias en la contabilidad en las empresas que comercializan en los mercados internacionales pueden generar distorsiones, si no se cuenta con un sistema contable homogéneo y avalado por los organismos internacionales en el ramo, lo que dificulta las decisiones empresariales al momento de comparar los estados financieros y tomar decisiones de inversión (Doupnik \& Perera, 2012).

2. El tipo de auditor. De acuerdo al argumento de Keryn Chalmers y Jayne M. Godfrey (2004), las grandes empresas procuran mantener un nivel alto de reputación mediante la búsqueda de ser auditadas por alguna de las firmas internacionales de auditoría de mayor prestigio, con el fin de cumplir los requerimientos de la normatividad contable. Las grandes firmas auditoras poseen mayor conocimiento de los 
estándares internacionales de contabilidad y, por lo tanto, proporcionan mayor nivel de certidumbre sobre los resultados de sus auditorías y sobre la calidad de la información revelada por las empresas analizadas. Este resultado se suma a los ya encontrados en estudios anteriores (Chalmers \& Godfrey, 2004; Lopes \& Rodrigues, 2007; Oliveira, Rodrigues \& Craig, 2006; Tsalavoutas, Evans \& Smith, 2010).

3. El tamaño de la firma. Este resultado es consistente con el de estudios analíticos
(Jensen \& Meckling, 1976) y empíricos (Chow \& Wong-Boren, 1987), que sustentan que las empresas más grandes tienen el incentivo de presentar mayor revelación financiera para incrementar la proporción de capital externo. Por otro lado, al ser el primer año de implementación de las IFRS en México de manera obligatoria, es posible sugerir que la revelación financiera de las grandes firmas fuera mayor por la motivación de mantener la demanda de inversionistas (Gray, Meek \& Roberts, 1995).

\begin{tabular}{|c|c|c|c|c|c|c|c|}
\hline $\begin{array}{c}\text { Variable } \\
\text { independiente }\end{array}$ & 1 & 2 & 3 & 4 & $4 a$ & 5 & $5 a$ \\
\hline \multirow[t]{2}{*}{ Ln de activos } & 0,3164 & 0,3350 & 0,2565 & 0,0497 & 0,0075 & & \\
\hline & $(2,52)^{* *}$ & $(2,77)^{* * *}$ & $(1,85)^{*}$ & $(0,28)$ & $(0,57)$ & & \\
\hline \multirow[t]{2}{*}{ Ln de ventas } & & & & & & $-0,0121$ & 0,0027 \\
\hline & & & & & & $(-0,08)$ & $(0,23)$ \\
\hline \multirow[t]{2}{*}{ Tipo de auditor } & 1,9160 & 2,0048 & 1,9506 & 1,9229 & 0,1263 & 1,9302 & 0,1277 \\
\hline & $(2,46)^{* * *}$ & $(2,61)^{* *}$ & $(2,62)^{* * *}$ & $(2,65)^{* * *}$ & $(2,82)^{* * *}$ & $(2,66)^{* * *}$ & $(2,83)^{* * *}$ \\
\hline \multirow[t]{2}{*}{ Apalancamiento } & $-0,1362$ & $-0,1296$ & $-0,1707$ & $-0,1723$ & $-0,0125$ & $-0,1679$ & $-0,0121$ \\
\hline & $(-0,57)$ & $(-0,53)$ & $(-0,74)$ & $(-0,86)$ & $(-0,89)$ & $(-0,84)$ & $(-0,87)$ \\
\hline \multirow[t]{2}{*}{ Anticipada } & & 0,6214 & 0,6942 & 0,2765 & 0,0196 & 0,2462 & 0,0164 \\
\hline & & $(1,24)$ & $(1,00)$ & $(0,47)$ & $(0,43)$ & $(0,41)$ & $(0,36)$ \\
\hline \multirow[t]{2}{*}{ Gobierno corporativo } & & 1,1179 & 1,0046 & 0,6011 & 0,0343 & 0,5006 & 0,0283 \\
\hline & & $(0,93)$ & $(0,80)$ & $(0,50)$ & $(0,38)$ & $(0,42)$ & $(0,32)$ \\
\hline \multirow[t]{2}{*}{$\begin{array}{l}\text { Cotización en el } \\
\text { extranjero }\end{array}$} & & & 0,5559 & 0,7715 & 0,0579 & 0,8382 & 0,0643 \\
\hline & & & $(0,96)$ & $(1,42)$ & $(1,39)$ & $(1,61)$ & $(1,62)$ \\
\hline \multirow[t]{2}{*}{ Sostenibilidad } & & & 1,1783 & 0,9785 & 0,0679 & 1,0172 & 0,0720 \\
\hline & & & $(1,10)$ & $(0,94)$ & $(0,86)$ & $(0,99)$ & $(0,93)$ \\
\hline \multirow[t]{2}{*}{ Multinacionalidad } & & & & 2,7451 & 0,1933 & 2,9215 & 0,2038 \\
\hline & & & & $(2,84)^{* * *}$ & $(2,62)^{* *}$ & $(2,78)^{* * *}$ & $(2,52)^{* *}$ \\
\hline $\begin{array}{l}\text { Número de } \\
\text { observaciones }\end{array}$ & & & & 93 & & & \\
\hline $\mathrm{R}^{2}$ ajustada & 0,1419 & 0,1582 & 0,1888 & 0,2514 & 0,2462 & 0,2507 & 0,2432 \\
\hline
\end{tabular}

Tabla 3

Resultados de mínimos cuadrados ordinarios con errores estándar robustos

Nota: * significativa al 10\%, ** al 5\% y *** al 1\%. Estadísticos t en paréntesis 


\section{Conclusiones}

Con datos de 2012 en el que las empresas mexicanas implementaron las IFRS, esta investigación valida y amplía el conocimiento de estudios previos que han analizado los factores que influyen en la revelación de instrumentos financieros en los reportes anuales de las compañías.

Esta investigación tiene particular importancia por ser la primera en revisar los reportes anuales de empresas que cotizan en la Bolsa Mexicana de Valores en el primer año en que adoptaron las IFRS y analizar el nivel de cumplimiento de este cuerpo normativo que alcanzaron. En el aspecto más técnico, contribuye de manera significativa en la evaluación de las empresas al calificar a cada una de acuerdo con los puntos que debe cumplir de conformidad con la IFRS 7: Instrumentos Financieros información a revelar. En este proceso, se identifican siete categorías de información a revelar, después de identificar los 26 requerimientos de mayor importancia señalados en la norma. Estas categorías permiten identificar los requisitos con mayor y menor grado de cumplimiento y también representan una contribución a un análisis más eficiente de la norma.

La investigación además de medir el índice de revelación de acuerdo con la metodología usada en trabajos anteriores, también lo hace con la técnica de componentes principales, lo que contribuye a una forma más integral sobre la manera de explotar la heterogeneidad existente en las categorías de revelación que cumplen las empresas analizadas. El empleo de esta metodología constituye una contribución significativa a la investiga- ción sobre índices de revelación de información financiera.

Los resultados sugieren que factores como el tipo de firma que audita los estados financieros de las empresas (firmas denominadas Big 4), el índice de multinacionalidad (medido por las ventas al extranjero como proporción de sus ventas totales) y, en menor medida, el tamaño de las empresas (medido por su nivel de activos y ventas) son las características que distinguen a aquellas empresas que presentan un índice de revelación mayor. Los resultados econométricos también sugieren lo que no resulta significativo para que una empresa cumpla en mayor medida lo señalado por el IASB, como puede ser su nivel de apalancamiento, su cotización en el extranjero, su adopción anticipada de las IFRS, o que revele información sobre sostenibilidad o gobierno corporativo.

Estos hallazgos son de interés para las empresas, organismos reguladores, emisores de normatividad y el medio académico. El organismo regulador, en este caso la CNBV, podría evaluar el avance de las empresas en el cumplimiento de adopción de las IFRS; e implementar mecanismos que contribuyan en la aplicación eficiente de esa normatividad. Por otro lado, organismos emisores de normas como el IASB y el CINIF ${ }^{3}$ pueden identificar cuáles son los requisitos más difíciles de cumplir y contribuir con capacitación y mejoras a la norma. En el caso del CINIF, tiene un insumo importante de información para valorar los requisitos que les va a solicitar a las pequeñas y medianas empresas del país, que aún seguirán

3 CINIF: Consejo Mexicano de Normas de Información Financiera. 
usando las normas contables mexicanas emitidas por este organismo.

Esta investigación nos invita a ampliar el trabajo realizado y abre una agenda de investigación que podría incluir la realización de un estudio con datos tipo panel, con la misma muestra de empresas pero incluyendo los reportes anuales de más años, y así evaluar el desempeño de las empresas al tener más experiencia en implementar las IFRS. Otra aportación en este sentido sería explorar los resultados considerando las empresas del sector financiero y analizar si las variables determinantes son las mismas a las de nuestros hallazgos, o hay otros determinantes para este sector en específico. Si bien presentamos estadísticas descriptivas por sector económico de nuestra muestra de empresas, otro trabajo futuro puede analizar si hay efectos diferenciados al separar la información por sector económico, considerando, al menos, los sectores más representativos para las empresas mexicanas.

\section{Referencias}

Abd-Elsalam, Omneya H. \& Weetman, Pauline (2003). Introduction International Accounting Standards to an Emerging Capital Market: Relative Familiarity and Language Effect in Egypt. Journal of International Accounting, Auditing \& Taxation, 12, 63-84. Disponible en: https://www.researchgate.net/ publication/222834253_Introducing_ International_Accounting_Standards_to_ an_emerging_capital_market_Relative_ familiarity_and_language_effect_in_Egypt
Bebbington, Jan; Larrinaga-González, Carlos \& Moneva-Abadía, José M. (2008). Corporate Social Reporting and Reputation Risk Management. Accounting, Auditing \& Accountability Journal, 21 (3), 337-361.

Disponible en: http://citeseerx.ist.psu.edu/ viewdoc/download?doi=10.1.1.178.8369\&re $\mathrm{p}=$ rep1\&type $=\mathrm{pdf}$

Bodnar, Gordon M. \& Gebhardt, Günther (1999). Derivatives Usage in Risk Management by US and German Nonfinancial Firms: A Comparative Survey. Journal of International Financial Management and Accounting, 10 (3), 153-187.

Cahan, Steven F. (1992). The Effect of the Antitrust Investigations on Discretionary Accruals: A Refined Test of the PoliticalCost Hypothesis. The Accounting Review, 67 (1), 77-95.

Cerf, Alan Robert (1961). Corporate Reporting and Investment Decisions. Berkeley: University of California Press, Institute of Business and Economic Research, IBER. Chalmers, Keryn \& Godfrey, Jayne M. (2004). Reputation Costs: The Impetus for Voluntary Derivative Financial Instrument Reporting. Accounting, Organizations and Society, 29 (2), 95-125.

Chambers, Dennis; Linsmeier, Thomas J.;

Shakespeare, Catherine \& Sougiannis, Theodore (2007). An Evaluation of SFAS No. 130 Comprehensive Income Disclosure. Review of Accounting Studies, 12 (4), 557-593.

Chow, Chee W. \& Wong-Boren, Adrian (1987). Voluntary Financial Disclosure by Mexican Corporations. The Accounting Review, 62 (3), 533-541. 
Chua, Wai Fong \& Taylor, Stephen L. (2008). The Rise and Rise of IFRS: An Examination of IFRS Diffusion. Journal of Accounting and Public Policy, 27 (6), 462-473.

Comisión Nacional Bancaria y de Valores, CNBV (2008). Boletín de prensa 56/2008. Disponible en: http://www.cnbv.gob. $\mathrm{mx} / \mathrm{SECTORES}$-SUPERVISADOS/ BURS\%C3\%81TIL/Boletines-de-Prensa/ Prensa\%20\%20Casas\%20de\%20 Bolsa/11\%20DE\%20NOVIEMBRE.pdf Cooke, Terry E. (1989). Voluntary Corporate Disclosure by Swedish Companies. Journal of International Financial Management and Accounting, 1 (2), 171-195.

Cooke, Terry E. (1991). An Assessment of Voluntary Disclosure in the Annual Reports of Japanese Corporations. The International Journal of Accounting, 26 (3), 174-189.

Cooke, Terry E. (1992). The Impact of Size, Stock Market Listing and Industry Type on Disclosure in the Annual Reports of Japanese Listed Corporations. Accounting and Business Research, 22 (87), 229-237.

Cotter, Julie; Lokman, Norziana \& Najah, Mutfah M. (2011). Voluntary Disclosure Research: Which Theory is Relevant? The Journal of Theoretical Accounting Research, 6 (2), 77-95.

Dechow, Patricia M.; Myers, Linda A. \& Shakespeare, Catherine (2010). Fair Value Accounting and Gains from Asset Securitizations: A Convenient Earnings Management Tool with Compensation Side-Benefits. Journal of Accounting and Economics, 49 (1-2), 2-25.
Deloitte (s.f.). IAS Plus. Disponible en: http:// www.iasplus.com/en/standards/ias/ias32

Devalle, Alain \& Rizzato, Fabio (2012). The Quality of Mandatory Disclosure: The Impairment of Goodwill. An Empirical Analysis of European Listed Companies. Procedia Economics and Finance, 2, 101-108. Doupnik, Timothy \& Perera, Hector (2012). International Accounting. 3a ed., Singapore: McGraw-Hill.

Doupnik, Timothy \& Salter, Stephen B. (1995). External Environment, Culture and Accounting Practice: A Preliminary Test of a General Model of International Accounting Development. The International Journal of Accounting, 30 (3), 189-206.

Everitt, Brian S. \& Dunn, Graham (2001). Applied Multivariate Data Analysis. London: Edward Arnold.

Fechner, Harry \& Kilgore, Alan (1994). The Influence of Culture Factors on Accounting Practice. The International Journal of Accounting, 29 (3), 265-277.

Fombrun, Charles J.; Gardberg, Naomi A. \& Barnett, Michael L. (2000). Opportunity Platforms and Safety Nets: Corporate Citizenship and Reputational Risk. Business and Society Review, 105 (1), 85-106. Disponible en: http://www.mv.helsinki.fi/ home/aula/Top20/fombrunetal2002.pdf

García-Fernández, Roberto; López-Díaz, Antonio \& González-Díaz, Belén (2008). Análisis económico y financiero de las empresas públicas estatales. Efectos del programa de modernización de 1996. Revista Galega de Economía, 17 (2), 1-24. Disponible en: https://www.researchgate. 
net/publication/26604592_Analisis_ economico_y_financiero_de_las_ empresas_publicas_estatales_Efectos_del_ programa_de_modernizacion_de_1996

Gebhardt, Günther (2012). Financial Instruments in Non-Financial Firms: What do We Know? Accounting and Business Research, 42 (3), 267-289.

Glaum, Martin \& Street, Donna L. (2003). Compliance with the Disclosure Requirements of Germany's New Market: IAS versus US GAAP. Journal of International Financial Management and Accounting, 14 (1), 64-100.

Gray, Sidney J.; Meek, Gary K. \& Roberts, Clare B. (1995). International Capital Market Pressures and Voluntary Annual Report Disclosures by US and UK Multinationals. Journal of International Financial Management and Accounting, 6 (1), 43-68.

Gujarati, Damobar N. \& Porter, Dawn C. (2010). Econometría. 5 ed., México: McGraw-Hill.

Haniffa, Roszaini \& Cooke, Terry E. (2002). Culture, Corporate Governance and Disclosure in Malaysian Corporations. Abacus, 38 (3), 317-349.

Hossain, Mahmud; Tan, Lin Mei \& Adams, Mike (1994). Voluntary Disclosure in an Emerging Capital Market; Some Empirical Evidence from Firms Listed on the Kuala Lumpur Stock Exchange. The International Journal of Accounting, 29 (3), 334-351.

Hotelling, Harold (1933). Analysis of Complex Statistical Variables into Principal Components. Journal of Educational Psychology, 24 (7), 498-520.
International Financial Reporting Standards Foundation, IFRS Foundation (s.f.). IFRS Use around the World. Disponible en: http:// www.ifrs.org/Use-around-the-world/Pages/ Jurisdiction-profiles.aspx

International Financial Reporting Standards Foundation, IFRS Foundation (2014). Normas Internacionales de Información Financiera. United Kingdom: IASB. Jensen, Michael C. \& Meckling, William H. (1976). Theory of the Firm: Managerial Behavior, Agency Costs and Ownership Structure. Journal of Financial Economics, 3 (4), 305-360. Disponible en: http:// ac.els-cdn.com/0304405X7690026X/1s2.0-0304405X7690026X-main.pdf?_ tid=199734e2-099b-11e7-855d-00000aab0f 02\&acdnat $=1489594921 \_0 f 5 f 7 e 0 a d 0 b 6 d a 0$ b3857c42d6b07092a

King, Ronald; Pownall, Grace \& Waymire, Gregory (1990). Expectations Adjustments Via Timely Management Forecasts: Review, Synthesis, and Suggestions for Future Research. Journal of Accounting Literature, 9, 113-144. Disponible en: https://www. researchgate.net/publication/246134066_ Expectations_adjustment_via_timely_ earnings_forecast_disclosure

Lang, Mark \& Lundholm, Russell (1993).

Cross Sectional Determinants of Analysis Ratings of Corporate Disclosures. Journal of Accounting Research, 31 (2), 246-271.

Lins, Karl V.; Servaes, Henri \& Tamayo, Ane (2011). Does Fair Value Reporting Affect Risk Management? International Survey Evidence. Financial Management, 40 (3), 
525-551. Disponible en: http://faculty. london.edu/hservaes/fm2011.pdf Lopes, Patrícia Teixeira \& Rodrigues, Lúcia Lima (2007). Accounting for Financial Instruments: An Analysis of the Determinants of Disclosure in the Portuguese Stock Exchange. The International Journal of Accounting, 42, 25-56. Disponible en: https://www.researchgate.net/ publication/4739828_Accounting_for_ financial_instruments_An_analysis_of_ the_determinants_of_disclosure_in_the_ Portuguese_stock_exchange

México (2014). Ley del Mercado de Valores, LMV. Diario Oficial de la Federación de los Estados Unidos Mexicanos, Distrito Federal, México, 10 de enero de 2014. Disponible en: http://www.bmv.com.mx/, http://www. diputados.gob.mx/LeyesBiblio/pdf/LMV.pdf Oliveira, Lídia; Rodrigues, Lúcia Lima \& Craig, Russell (2006). Firm-Specific Determinants of Intangibles Reporting: Evidence from the Portuguese Stock Market. Journal of Human Resource Costing \& Accounting, 10 (1), 11-33. Disponible en: https://www.researchgate. net/publication/235269474_Firm-specific_ determinants_of_intangibles_reporting Evidence_from_the_Portuguese_stock_ market

Popova, Tatiana; Georgakopoulos, Georgios; Sotiropoulos, Ioannis \& Vasileiou, Konstantinos Z. (2013). Mandatory Disclosure and Its Impact on the Company Value. International Business Research, 6 (5), 1-16. http://ccsenet.org/journal/index.php/ ibr/article/view/26651/16277
Programa de las Naciones Unidas para el Desarrollo, PNUD (2014). Informe sobre Desarrollo Humano 2014: Sostener el progreso humano: reducir vulnerabilidades y construir resiliencia. Nueva York: Programa de las Naciones Unidas para el Desarrollo, PNUD. Disponible en: http://www.undp. org/content/undp/es/home/librarypage/ hdr/2014-human-development-report.html Rescala, Carmen; Devincenzi, Gustavo; Rohde, Gricela; Bonaffini, María Liliana; Giraudo, Marta V.; Bernaola, Gustavo \& Pavón, Rita (2012). Dos modelos para determinar la eficiencia de una empresa constructora. Tordesillas Revista de Investigación Multidisciplinar, TRIM, 5, 21-38. Disponible en: http://www5.uva.es/trim/TRIM/ TRIM5_files/TRIM5.pdf, http://www5.uva. es/trim/TRIM/TRIM5_files/MODELOS.pdf

Ross, Stephen A. (1977). The Determination of Financial Structure: The IncentiveSignalling Approach. Bell Journal of Economics, 8 (1), 23-40.

Song, Chang Jong; Thomas, Wayne B. \& Yi, Han (2010). Value relevance of FAS No. 157 Fair Value Hierarchy Information and the Impact of corporate Governance Mechanisms. The Accounting Review, 85 (4), 1375-1410.

Street, Donna L. \& Bryant, Stephanie M. (2000). Disclosure Level and Compliance with IASs: A Comparison of Companies with and without US Listings and Fillings. The International Journal of Accounting, 35 (3), 305-329. Disponible en: https://www.researchgate. net/publication/4739418_Disclosure_ 
Level_and_Compliance_with_IASs_A_ Comparison_of_Companies_With_and_ Without_US_Listings_and_Filings

Street, Donna L. \& Gray, Sidney J. (2001). Observance of International Accounting Standards: Factors Explaining Noncompliance. Association of Chartered Certified Accountants, ACCA, ACCA Research Report, 74, London.

Tarca, Ann; Morris, Richard D. \& Moy, Melissa (2005). An Investigation of the Relationship between Use of International Accounting Standards and Source of Company Finance in Germany, EAA. Gothenburg: Ponencia, XXVIII Congreso Anual de la European Accounting Association. Disponible en: https://papers.ssrn.com/sol3/papers. cfm?abstract_id $=773807$

Toscano-Moctezuma, Juan Alfonso \& GarcíaBenau, María Antonia (2012). Estrategias de las cuatro grandes firmas de auditoría en México. Revista Europea de Dirección y Economía de la Empresa, 20 (1), 89-104. Disponible en: https://dialnet.unirioja.es/ descarga/articulo/3416136.pdf

Tsalavoutas, Ioannis; Evans, Lisa \& Smith, Mike (2010). Comparison of Two Methods for Measuring Compliance with IFRS Mandatory Disclosure Requirements. Journal of Applied Accounting Research, 11 (3), 213-228.

United Nations Conference on Trade and Development, UNCTAD, Intergovernmental Working Group of Experts on International Standards of Accounting and Reporting, ISAR (2014). Key Foundations for High-Quality Corporate
Reporting: Good Practices of Monitoring and Enforcement, and Compliance Mechanisms. Disponible en: http://unctad.org/meetings/ en/SessionalDocuments/ciiisard70_en.pdf

Vázquez-Alfonso, Yasser; Guerra-Bustillo, Caridad Walkiria \& Sánchez-León, Orlando Enrique (2014). Modelación estadístico-computacional para el estudio de la sostenibilidad socioeconómica de la empresa pecuaria Valle del Perú. Revista Investigación Operacional, 35 (2), 121-129. Disponible en: https://rev-inv-ope.univparis1.fr/IMG/pdf_35214-04.pdf

Verrecchia, Robert E. (1983). Discretionary Disclosure. Journal of Accounting and Economics, 5, 179-194.

Wallace, R. S. Olusegun \& Naser, Kamal (1995). Firm-specific Determinants of Comprehensiveness of Mandatory Disclosure in the Corporate Annual Reports of Firms on the Stock Exchange of Hong Kong. Journal of Accounting and Public Policy, 14 (4), 311-368.

Watts, Ross L. \& Zimmerman, Jerold L. (1978). Towards a Positive Theory of the Determination of Accounting Standards. The Accounting Review, 53 (1), 112-134. Disponible en: http://jpkc.sysu.edu.cn/kjll/ wenxian/12.pdf

Watts, Ross L. \& Zimmerman, Jerold L. (1986). Positive Accounting Theory. Englewood Cliffs, New Jersey: Prentice Hall.

Watts, Ross L. \& Zimmerman, Jerold L. (1990). Positive Accounting Theory: A Ten Year Perspective. The Accounting Review, 65 (1), 131-156. 
Zaidi, Syed \& Paz, Veronica (2015). The Impact of IFRS Adoption: A Literature Review. Journal of Theoretical Accounting Research, 10 (2), 116-141.

Zarzeski, Marilyn Taylor (1996). Spontaneous Harmonization Effects of Culture and Market Forces on Accounting Disclosure Practices. Accounting Horizons, 10, 18-37. Zehri, Fatma \& Chouaibi, Jamel (2013).

Adoption Determinants of the International Accounting Standards IAS/IFRS by the Developing Countries. Journal of Economics, Finance, and Administrative Science, 18, 5662. Disponible en: http://jefas.esan.edu.pe/ index.php/jefas/article/view/270/256 Zhang, Haiwen (2009). Effect of Derivative Accounting Rules on Corporate Risk-
Management Behavior. Journal of Accounting and Economics, 47 (3), 244-264.

- Fecha de recepción: 15 de junio de 2016

- Fecha de aceptación: 8 de agosto de 2016

- Disponible en línea: 14 de diciembre de 2016

\section{Para citar este artículo}

Vásquez-Quevedo, Noemí \& Mora-Rivera, José Jorge (2016). Análisis de los determinantes de revelación de instrumentos financieros mediante el uso de componentes principales: el caso de empresas mexicanas. Cuadernos de Contabilidad, 17 (44), 467-492. https://doi.org/10.11144/ Javeriana.cc17-44.adri 
ANÁLISIS DE LOS DETERMINANTES DE REVELACIÓN DE INSTRUMENTOS FINANCIEROS / N. VÁSQUEZ, J. MORA / 489

Anexo 1
Empresas seleccionadas

\begin{tabular}{|c|c|c|c|}
\hline Accel, SAB de CV & Dine, $\mathrm{SAB}$ de $\mathrm{CV}$ & $\begin{array}{l}\text { Grupo Famsa, SAB } \\
\text { de CV }\end{array}$ & Ingeal, $\mathrm{SAB}$ de $\mathrm{CV}$ \\
\hline Alfa, SAB de CV & Edoardos Martin, SAB de CV & $\begin{array}{l}\text { Grupo Gigante, SAB } \\
\text { de CV }\end{array}$ & $\begin{array}{l}\text { Inmuebles Carso, } \mathrm{SAB} \text { de } \mathrm{CV} \text {, } \\
\text { Incarso }\end{array}$ \\
\hline Alpek, SAB de CV & $\begin{array}{l}\text { El Puerto de Liverpool, SAB } \\
\text { de CV }\end{array}$ & $\begin{array}{l}\text { Grupo Herdez, SAB } \\
\text { de CV }\end{array}$ & $\begin{array}{l}\text { Internacional de Cerámica, } \\
\mathrm{SAB} \text { de } \mathrm{CV}\end{array}$ \\
\hline Alsea, SAB de CV & $\begin{array}{l}\text { Empresas Cablevisión, SA } \\
\text { de CV }\end{array}$ & $\begin{array}{l}\text { Grupo Industrial } \\
\text { Maseca, SAB de CV, } \\
\text { GIMSA }\end{array}$ & $\begin{array}{l}\text { Kimberly-Clark de México, } \\
\text { SAB de CV }\end{array}$ \\
\hline $\begin{array}{l}\text { Altos Hornos de México, SAB } \\
\text { de CV, AHMSA }\end{array}$ & Empresas ICA, SAB de CV & $\begin{array}{l}\text { Grupo Industrial } \\
\text { Saltillo, SAB de CV, GIS }\end{array}$ & $\begin{array}{l}\text { Maxcom Telecomunicaciones, } \\
\text { SAB de CV, Maxcom }\end{array}$ \\
\hline América Móvil, SAB de CV & $\begin{array}{l}\text { Farmacias Benavides, SAB } \\
\text { de CV }\end{array}$ & Grupo Kuo, SAB de CV & Médica Sur, SAB de CV \\
\hline $\begin{array}{l}\text { Arca Continental, SAB de CV, } \\
\text { ArcaContal }\end{array}$ & $\begin{array}{l}\text { Fomento Económico } \\
\text { Mexicano, SAB de CV, } \\
\text { FEMSA }\end{array}$ & $\begin{array}{l}\text { Grupo Lamosa, SAB } \\
\text { de CV }\end{array}$ & $\begin{array}{l}\text { Megacable Holdings, SAB } \\
\text { de CV }\end{array}$ \\
\hline Axtel, SAB de CV & G Collado, SAB de CV & $\begin{array}{l}\text { Grupo Mexicano de } \\
\text { Desarrollo, SAB, GMD }\end{array}$ & Mexichem, SAB de CV \\
\hline Bio Pappel, SAB de CV & $\begin{array}{l}\text { Genomma Lab Internacional, } \\
\text { SAB de CV }\end{array}$ & $\begin{array}{l}\text { Grupo México, SAB } \\
\text { de CV }\end{array}$ & Minera Frisco, $\mathrm{SAB}$ de $\mathrm{CV}$ \\
\hline Cemex, SAB de CV & GMD Resorts, SAB de CV & $\begin{array}{l}\text { Grupo Minsa, SAB } \\
\text { de CV }\end{array}$ & $\begin{array}{l}\text { Nutrisa, SAB de CV (deslistada } \\
\text { en 2014) }\end{array}$ \\
\hline CMR, SAB de CV & Gruma, SAB de CV & $\begin{array}{l}\text { Grupo Modelo, SAB } \\
\text { de CV }\end{array}$ & Ohl México, SAB de CV \\
\hline Coca-Cola Femsa, SAB de CV & Grupe, SAB de CV & $\begin{array}{l}\text { Grupo Palacio de } \\
\text { Hierro, SAB de CV }\end{array}$ & $\begin{array}{l}\text { Organización Soriana, SAB } \\
\text { de } \mathrm{CV}\end{array}$ \\
\hline $\begin{array}{l}\text { Compañía Minera Autlán, } \\
\text { SAB de CV }\end{array}$ & $\begin{array}{l}\text { Grupo Aeroméxico, SAB } \\
\text { de } \mathrm{CV}\end{array}$ & $\begin{array}{l}\text { Grupo Pochteca, SAB } \\
\text { de CV }\end{array}$ & $\begin{array}{l}\text { Promotora Ambiental, SAB } \\
\text { de } \mathrm{CV}\end{array}$ \\
\hline Consorcio Ara, SAB de CV & $\begin{array}{l}\text { Grupo Aeroportuario del } \\
\text { Centro Norte, SAB de CV }\end{array}$ & $\begin{array}{l}\text { Grupo Posadas, SAB } \\
\text { de } C V\end{array}$ & $\begin{array}{l}\text { Promotora y Operadora de } \\
\text { Infraestructura, SAB de CV, } \\
\text { PINFRA }\end{array}$ \\
\hline Consorcio Aristos, SAB de CV & $\begin{array}{l}\text { Grupo Aeroportuario del } \\
\text { Pacífico, SAB de CV, GAP }\end{array}$ & $\begin{array}{l}\text { Grupo Radio Centro, } \\
\text { SAB de CV }\end{array}$ & Proteak Uno, SAB de CV \\
\hline Consorcio Hogar, SAB de CV & $\begin{array}{l}\text { Grupo Aeroportuario del } \\
\text { Sureste, SAB de CV }\end{array}$ & $\begin{array}{l}\text { Grupo Simec, SAB de } \\
\text { CV }\end{array}$ & Rassini, SAB de CV \\
\hline $\begin{array}{l}\text { Controladora Comercial } \\
\text { Mexicana, SAB de CV }\end{array}$ & Grupo Bafar, SAB de CV & $\begin{array}{l}\text { Grupo Sports World, } \\
\text { SAB de CV }\end{array}$ & Sare Holding, SAB de CV \\
\hline $\begin{array}{l}\text { Convertidora Industrial, SAB } \\
\text { de CV, Conver }\end{array}$ & Grupo Bimbo, SAB de CV & Grupo Televisa, SAB & Tv Azteca, SAB de CV \\
\hline Corporación Geo, SAB de CV & Grupo Carso, SAB de CV & Grupo Vasconia, SAB & $\begin{array}{l}\text { Urbi Desarrollos Urbanos, SAB } \\
\text { de CV }\end{array}$ \\
\hline $\begin{array}{l}\text { Corporación Interamericana } \\
\text { de Entretenimiento, SAB de } \\
\text { CV, CIE }\end{array}$ & Grupo Casa Saba, SAB de CV & $\begin{array}{l}\text { Hilasal Mexicana, SAB } \\
\text { de CV }\end{array}$ & Vitro, SAB de CV \\
\hline
\end{tabular}




\section{0 / VOL. 17 / NO. 44 / JULIO-DICIEMBRE 2016}

\begin{tabular}{llll}
\hline & & Impulsora del Desarrollo & \\
$\begin{array}{l}\text { Corporación Moctezuma, SAB } \\
\text { de CV }\end{array}$ & Grupo Cementos de & y el Empleo en América & WalMart de México, SAB de \\
& Chihuahua, SAB de CV & Latina, SAB de CV, & CV \\
IDEAL & Industrias Bachoco, SAB \\
Corporativo Fragua, SAB de & Grupo Collado, SA de CV & de CV \\
CV & Grupo Comercial Chedraui, & Industrias Ch, SAB de \\
Cydsa, SAB de CV & SAB de CV & CV, ICH \\
Desarrolladora Homex, SAB & Industrias Peñoles, SAB \\
de CV & Grupo Elektra, SAB de CV & de CV \\
\hline
\end{tabular}

Fuente: elaboración propia de acuerdo con información publicada en la Bolsa Mexicana de Valores: http://www.bmv.com.mx/ 


\section{Anexo 2 \\ Categorías y criterios}

\section{Categorías}

Categorías de instrumentos financieros (IF)

Valor razonable

Riesgos (generalidades)

Riesgo de liquidez

Riesgo de mercado

Estado de resultados integral

\section{Criterios}

Activos financieros (AF) medidos al valor razonable (VR) con cambios en resultados.

Pasivos financieros (PF) al VR con cambios en resultados, separando:

AF medidos al costo amortizado (CA).

$\mathrm{PF}$ medidos al costo amortizado (CA).

AF o PF al VR con cambios en resultados - Máximo nivel de exposición al riesgo de crédito del

AF.

VR de cada clase de AF y de PF.

Agrupar AF y PF en clases.

Para cada clase de IF, revelar métodos y técnicas de valoración.

Clasificar las mediciones a VR usando una jerarquía de VR.

Para las mediciones del VR, revelar en cada clase de IF el nivel de jerarquía.

Si el mercado de un IF no es activo, mencionar la técnica de medición para determinar el VR.

Las exposiciones al riesgo y la forma en que estas surgen.

Los objetivos, políticas y procesos para la gestión del riesgo, así como métodos usados para

medirlo.

Datos cuantitativos resumidos acerca de su exposición al riesgo al final del período.

Importe que mejor represente su máximo nivel de exposición al riesgo de crédito al final del período.

Información acerca de la calidad crediticia de los AF que no están en mora ni hayan deteriorado su valor.

Análisis de vencimiento para pasivos financieros derivados y no derivados.

Descripción de cómo se gestiona el riesgo de liquidez.

Análisis de sensibilidad para cada tipo de riesgo de mercado al que la entidad esté expuesta al final.

Métodos e hipótesis utilizados al elaborar el análisis de sensibilidad.

AF a VR con cambios en resultados.

PF a VR con cambios en resultados.

AF medidos al CA.

PF medidos al CA.

Importes totales de ingresos y gastos por interés.

Ingresos y gastos por comisiones.

Fuente: elaboración propia de acuerdo con la Norma Internacional de Información Financiera: IFRS 7 
492 / VOL. 17 / NO. 44 / JULIO-DICIEMBRE 2016

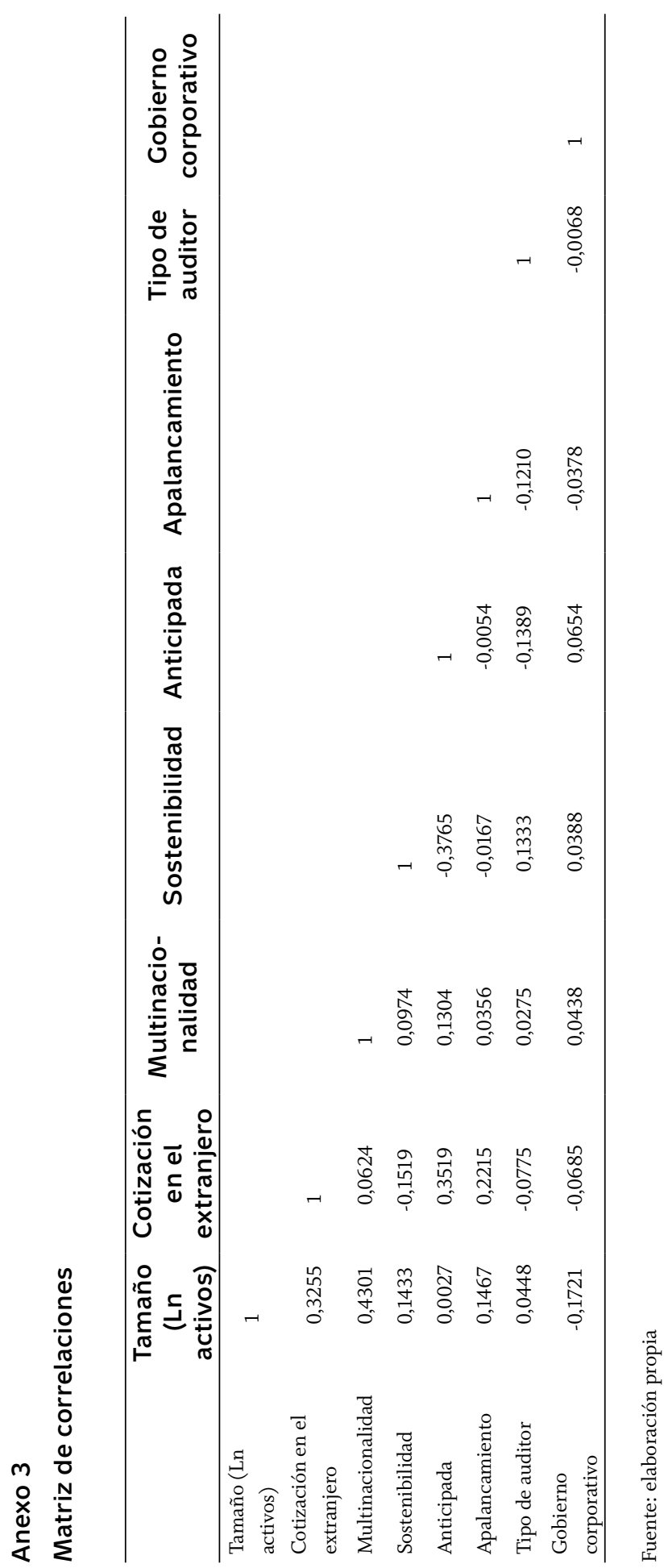

